\title{
Automatic Scheduling Method for Maintenance Outages Plan of Transmission System
}

\author{
Sabah M. Askar ${ }^{1} \&$ Fowzi Yousef Al-Somali ${ }^{2}$ \\ ${ }^{1}$ Gulf University, Kingdom of Bahrain \\ ${ }^{2}$ National GridSA, Khobar, Kingdom of Saudi Arabia \\ Correspondence: Sabah M. Asker, College of Engineering, Gulf University, Kingdom of Bahrain. Tel: \\ 973-3949-7879. E-mail: dr.sabah.mansou@gulfunivesity.net
}

\author{
Received: February 14, 2013 Accepted: February 26, $2013 \quad$ Online Published: April 11, 2013 \\ doi:10.5539/emr.v2n1p88 URL: http://dx.doi.org/10.5539/emr.v2n1p88
}

\begin{abstract}
The main objective of this paper is to develop a model for automatic scheduling of outages tasks for maintenances using Artificial Intelligent tool (Tabu Search). The developed method of TS is used to reduce maintenance outages for electrical power system with high standard of reliability. The developed method solved the maintenance outages with taking in consideration two main problems. The first one is power flow constraints and contingency studies constrains. The second problem is the number of the crews. The developed method for automatic scheduling of maintenance outage tasks using TS is systematically applied to reconfiguration of networks and work starting date scheduling. The works progress is maximized in the scheduling period while minimized the carry-over work to the next scheduling period. The developed method was used in 20-bus test system.
\end{abstract}

Keywords: artificial intelligent, automatic scheduling, contingency analysis, maintenance scheduling, outages plan, optimization of maintenance schedules, power flow, tabu search, transmission system

\section{Introduction}

Modern industrialized societies depend on the availability of a reliable supply of electricity to sustain their functions and standards of living. These expansions made the electric power systems and their operations the most complex systems of today's civilization, due to the highly nonlinear and computationally difficult environment involved (Furukawa et al., 1991). The planning and operation of these systems should therefore be as efficient, optimal as possible, with high reliability and with low costs. For these reasons, automatic scheduling method draws a lot a attentions.

Maintenance -scheduling optimization has been studied extensively in the open literature. Most of these studies and literature have been directly about or related to generation plants/units maintenance scheduling aiming to cut the fuel costs and obtaining good maintenance schedules. However, maintenance scheduling in transmission and distribution systems is occupying a small portion of the literature. This was the motivation to study this subject.

The objective of this paper is to develop a model for automatic scheduling of outages tasks for transmission and substation maintenance using Artificial Intelligent tool such as Tabu Search and to find the optimal solution for maintenance schedules to minimize the period of doing maintenance activity with preventing any carry-over of work activity to next week. Tabu search is chosen because of its ability to cross the boundaries of feasibility or local optimality, which were usually treated as barriers (Glover, Taillard, \& Werra, 1993; Glover, 1989).

There are two main problem to find optimal solution for maintenance schedules. First problem is the power flow and contingency analysis. The second problem is the number of crew or the number of work groups which responsible to execute the work (Reihani, Davodi, Najjar, \& Norouzizadeh, 2010; Alshaikh, \& El-Amin, 2001). The number of crew (qualified crew) is very important because some time there is conflict between two or more activities in some area not because the continuity of the power (power flow) but because the crews cannot do both or all activities in same time.

This paper is organized in five chapters. First chapter is introduction flowed by chapter two which addresses the problem explanation and problem constraints. The chapter three is method description. The results and discussion are given in chapter four. Chapter five represents the conclusions. 


\section{Problem Explanations and Problem Constraints}

Each division or department has one or more planners or maintenance schedulers. They are doing maintenance schedules for all equipment which related to their areas or divisions (Furukawa et al.,1991). The schedules are prepared weekly and consist of the starting date of the outages and the end date of the outages which include the duration of the outages. Also, the schedules contain the equipment's names, equipment's numbers and the kind of work which will be carried out. In other words, the initial schedules which requested by the planners do not contain any power flow study or contingency analysis and these schedules are sent to power dispatch (Alshaikh \& El-Amin, 2001; Yang, 2009).

The power dispatch centers receive all schedules (initial schedules or requested schedules) from all divisions so that the power dispatch planners can study these schedules from power flow point of view and contingency analysis (Yang, 2009; Tanaka, Magori, Niimura, \& Yokoyama, 2010). The developed method solved the maintenance outages with taking in consideration two main problems. The first is power flow constraints and contingency studies constrains. The second problem is the number of the crews which will be execute the work. In this case, the higher priority will be executed first and the activities with less priority will be moved to another days using Tabu Search method. If some of activities can not be done because of the shortage of the equlified man power. In this case also, the higher priority will be executed first and the activities with less priorities will be moved to another days using Tabu Search method (Reihani, Davodi, Najjar, \& Norouzizadeh, 2010; Alshaikh, \& El-Amin, 2001).

If the work starting dates are fixed and the incorporation of another task into the network causes an overloaded then network reconfiguration, regulation of dispatched power or a change of unit commitment may eliminate this overload (Sawa, 1999). Two possible network reconfiguration methods are visible. One is based on heuristic rules for the network pattern. The rules of this kind are acquired from expert operators in the load dispatch centers which are usually based on the mission of the company or on agreements with customers (Sawa, 1999). The other is based on TS. In this work, the overload elements are determined separately by using power world simulation program. After the overload determined as a list of conditions, then it became input in the main program and the program will build on it. In this case, the program will eliminate power flow constraints by avoiding these elements (or branches) to be out for maintenance in same time. Furthermore, when the program checks any schedule is free from overload, (it means) it checks these condition. Figure 1 shows the process of getting power flow constraints list. First, generate base configuration from system input data. This step includes building up the admittance matrix of the system which is considered to be base configuration (original configuration) of the network before incorporating any change (outages) requested by the maintenance centers. Second, study power flow for each case of contingency n-1 and the same for n-2 and n-3.After that, we make the list of power flow constraints to be input to the main TS program.

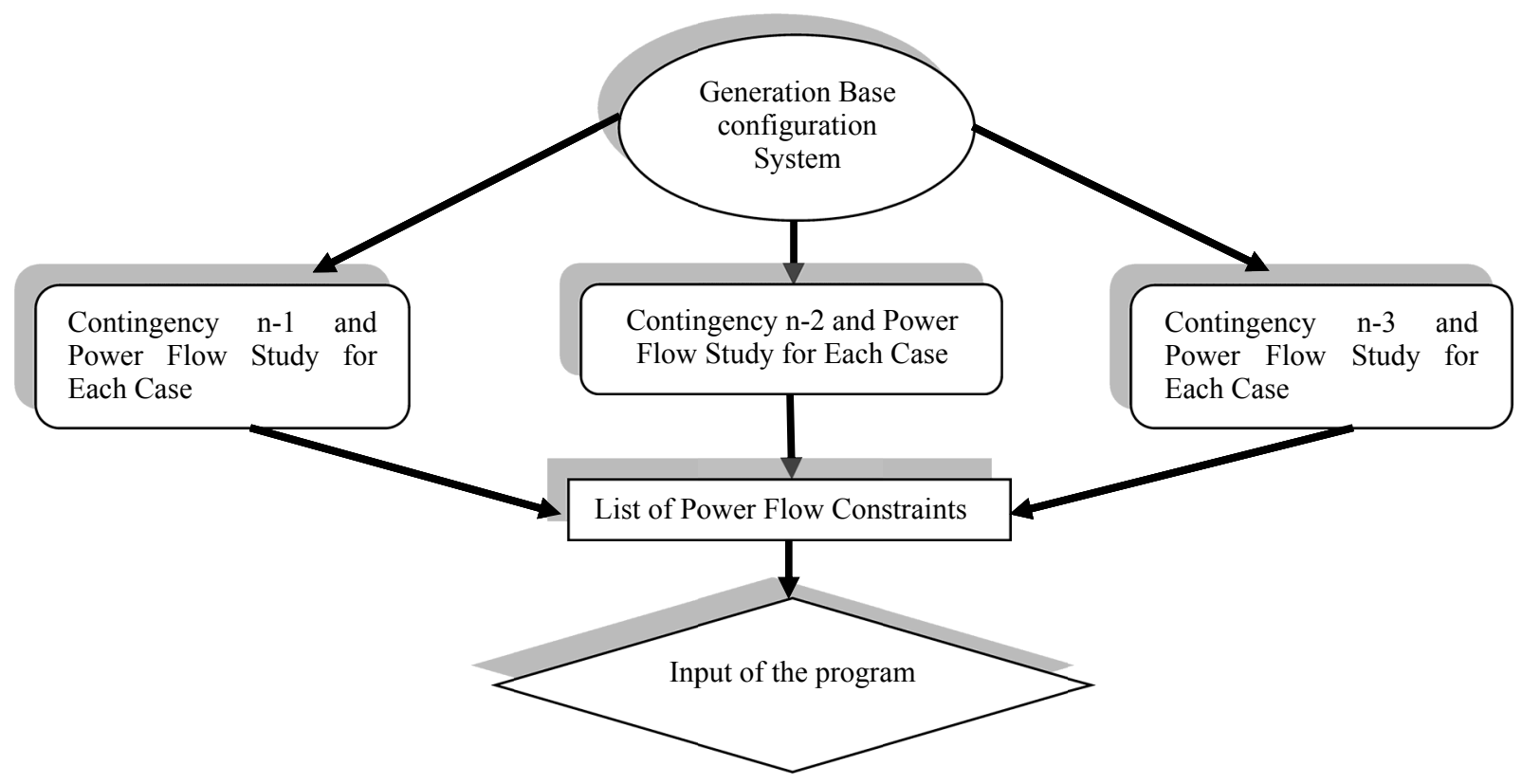

Figure 1. Block diagram of power flow constraints 


\section{Method Descriptions}

The Tabu Search is an iteration method used to solve the optimization problem and it was invented by Glover. TS starting with initial solution and search for optimal solutions in the neighborhood solutions and then compare between the neighborhood solutions. The best solution between the neighborhood solution will be save in memory then search for optimal solution to the obtained solution (even though it is worse than current solution). This process will be repeated (Glover, Taillard, \& Werra, 1993; Glover, 1989).

TS method is one of the combinatorial optimization methods and based on an efficient search with local optimum avoidance. With 'tabu list' of moves or states forbidden for a certain number of steps, the search is controlled to avoid cycling, covering a wide solution space and reaching diversified solutions. A basic move to a new solution is worse than the present solution- refer to figure 2. Therefore, the search speed is as fast as for a local search. The basic steps in TS method are as follows (Sawa, 1999):

Step1: Set the initial solution (supposed to be the best solution). Tabu list is empty since it was no created any step or move.

Step 2: Enumerate candidates (moves) which consists of the neighborhood of the current solution, exception of Tabu movement. These must be moves in the neighborhood of initial solution space.

Step 3: Go to the best candidate. Add a new move to list of Tabu moves and discard the oldes move from the list of Tabu list if the list of Tabu exceed a specified length (Tabu size list).

Step 4: Repeat steps 2 and 3 until it is satisfied the termination conditions (stop criteria).

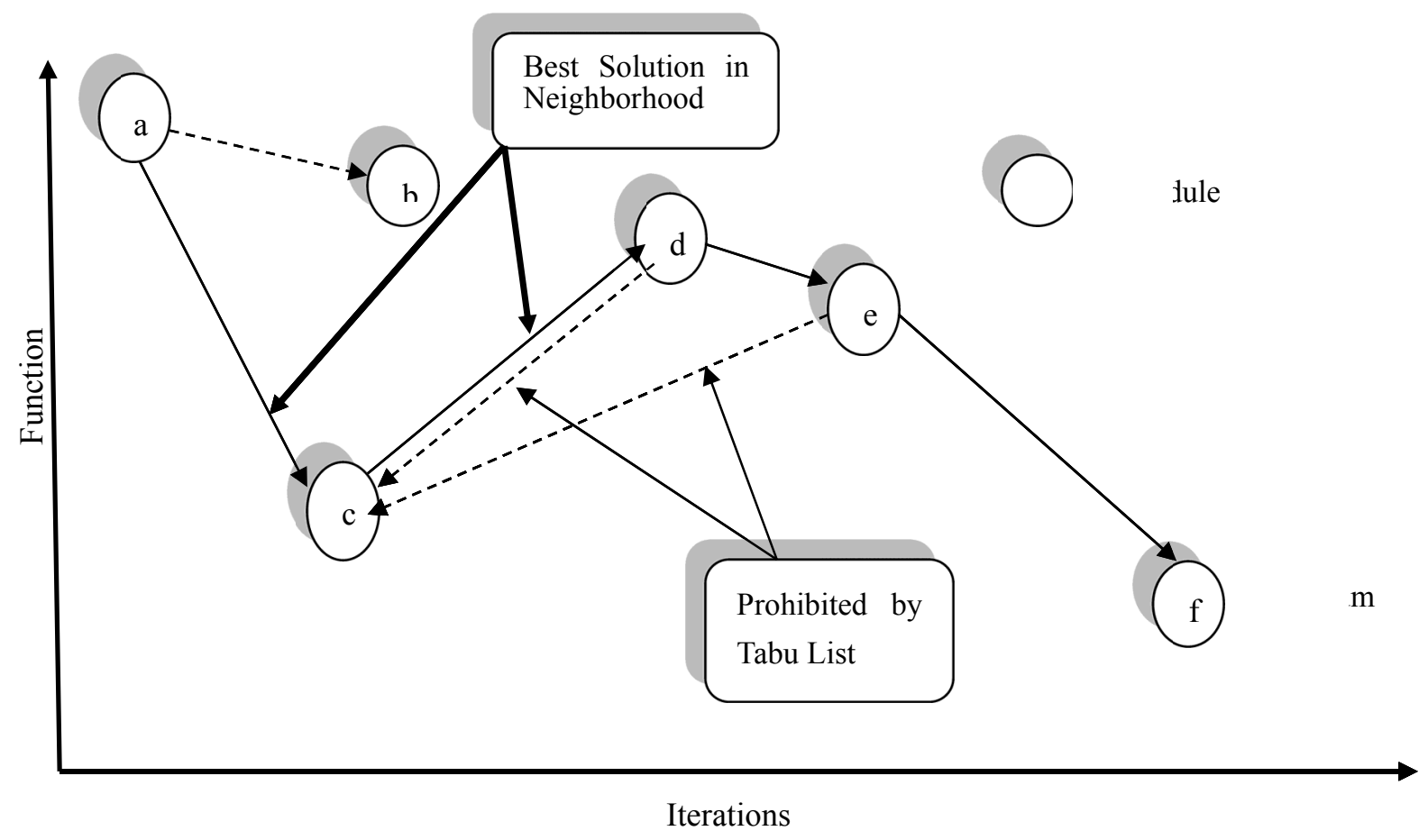

Figure 2. Tabu search implementation for maintenance scheduling of outages tasks

\section{Results \& Discussion}

The 20-Bus test system demonstrated in figure 3. It contains the following:

1. Network: $115 \mathrm{KV}, 13.8 \mathrm{KV}$ network

2. Network elements: 20 buses, 36 lines, 8 transformers, 7 generators.

3. Contingency study of n-1, n-2 and n-3 were applied on all of 20-bus system.

4. One week scheduling (7 day): 2012. 
5. Power flow will be checked at the demand peak and off-peak time daily.

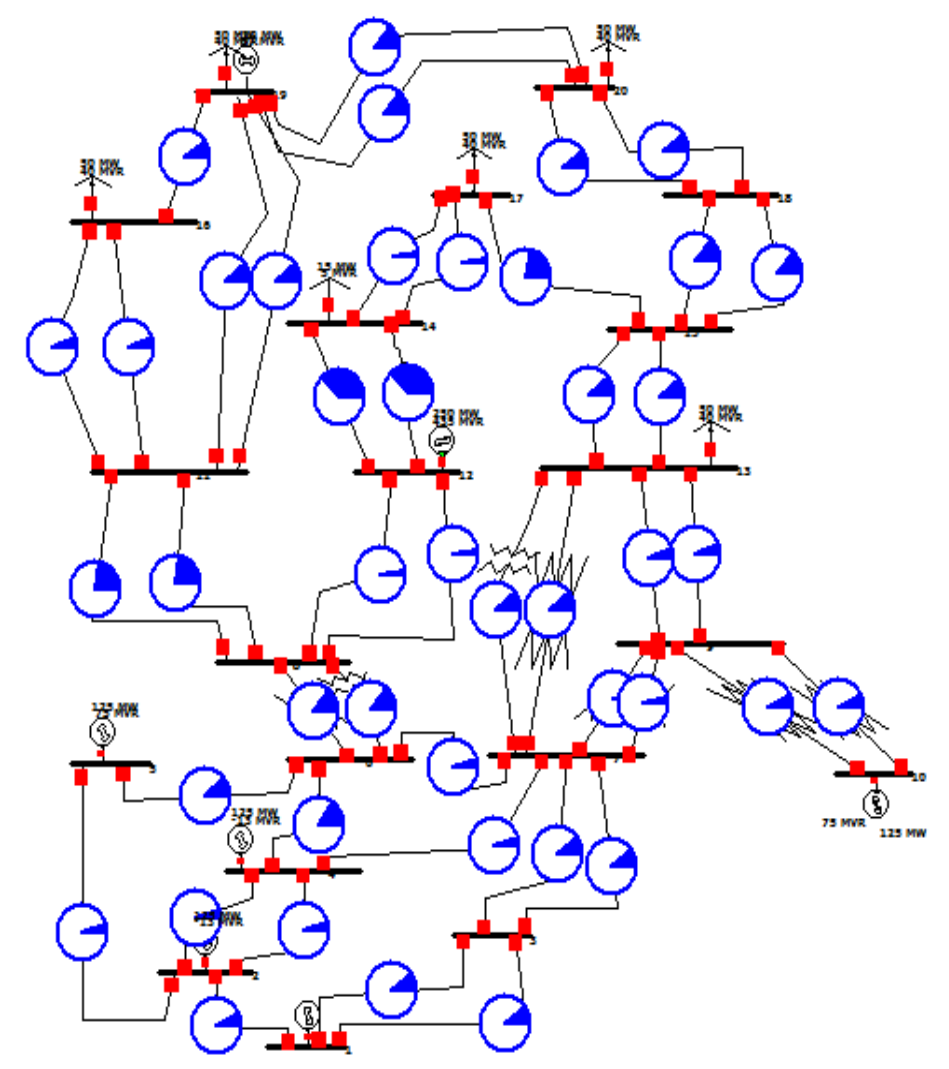

Figure 3. Base case of the test system

Power system are normally operated so that overload do not occur either in real-time or under any single outage (n-1) or complex outages (n-2).Contingency analysis (CA) is a "what if" scenario simulator that evaluates the impacts on an electric power system when problem occur (Stagg, \& El-Abiad, 1968; Internet page, http://smartgrid.epri.com ). For example, if line one taken out of the system then does it lead to overload. Furthermore, what impact this line on the system. This scenario called n-1 contingency. The n- 1 contingency is initial loss of a singal transmission line. Also, the $n-2$ contingency is a sequence of events consisting of the initial loss of a single transmission line, following another loss of a single transmission line (and so on for $n-3$ and $n-4$ contingencies). In the flowing steps the process of getting contingency analysis list. First, the base case should be free from any over load. Second, one line taken out of the system then power flow study should be done on the system. If there is any over load then this line should be listed in the contingency list. All lines should be taken out separately. Third, the same done on n-2 except that the two lines take out simultaneity. For example, line one out with line two then power flow done after that line one and line three taken outs together then power flow done and so on.

$\mathrm{n}-2$ (contingency) $=\{($ line 1 , line 2$),($ line 1, line 3$),($ line 1, line 4$) \ldots,($ line 2, line 3,(line 2, 4), (line 2, line 5)...,(line 3, line 4),(line 3, line 5)...

Where (line out, line out) simultaneity.

$\mathrm{n}-3=\{($ line 1, line 2, line 3$),($ line 1, line 2, line 4), (line 1, line 2, line 5$) \ldots$ (line 1, line 3, line 4), (line 1, line 3 , line4)...\}

Where $\{($ line out, line out, line out $)\}$ simultaneity.

Figure 3 shows the base case of the system before doing any contingency on the system. Two cases of power flow study done (as an example) by using Power World simulator 8.0 edition. Figure 4 shows first case which representing the $n-1$ case after doing power flow analysis (the line located between bus 15 and bus18). The analysis shows clearly that taken out this line will not affect any branches by overloading. In conclusion, this line 
will not be added to the contingency list. Taken two line simultaneity is the second case which is shown in figure 5 and it is represent $n-2$ after doing Power flow analysis on the system (both of the lines are located between bus 15 and bus 18). The analysis shows clearly that taken both of those lines simultaneity will lead to total black out of the system. So that, both of these two lines simultaneity be added in the contingency list.

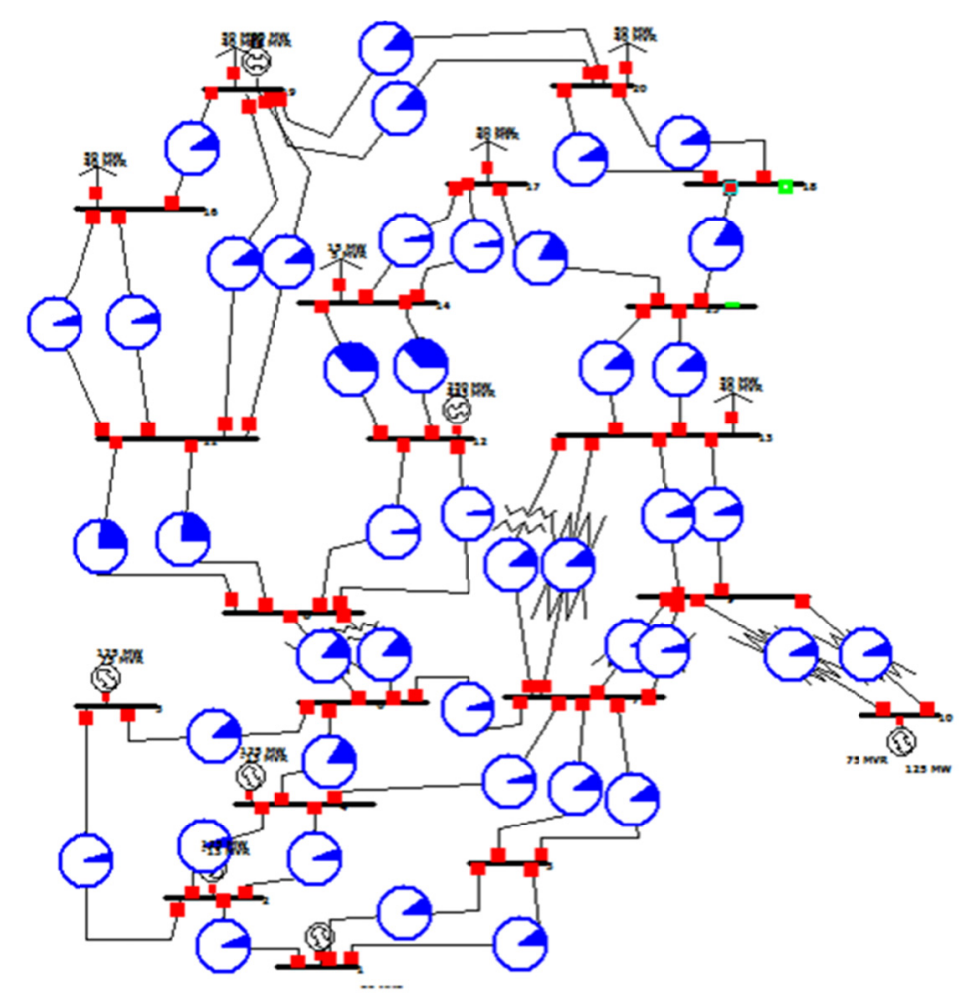

Figure 4. Represents N-1 power flow case

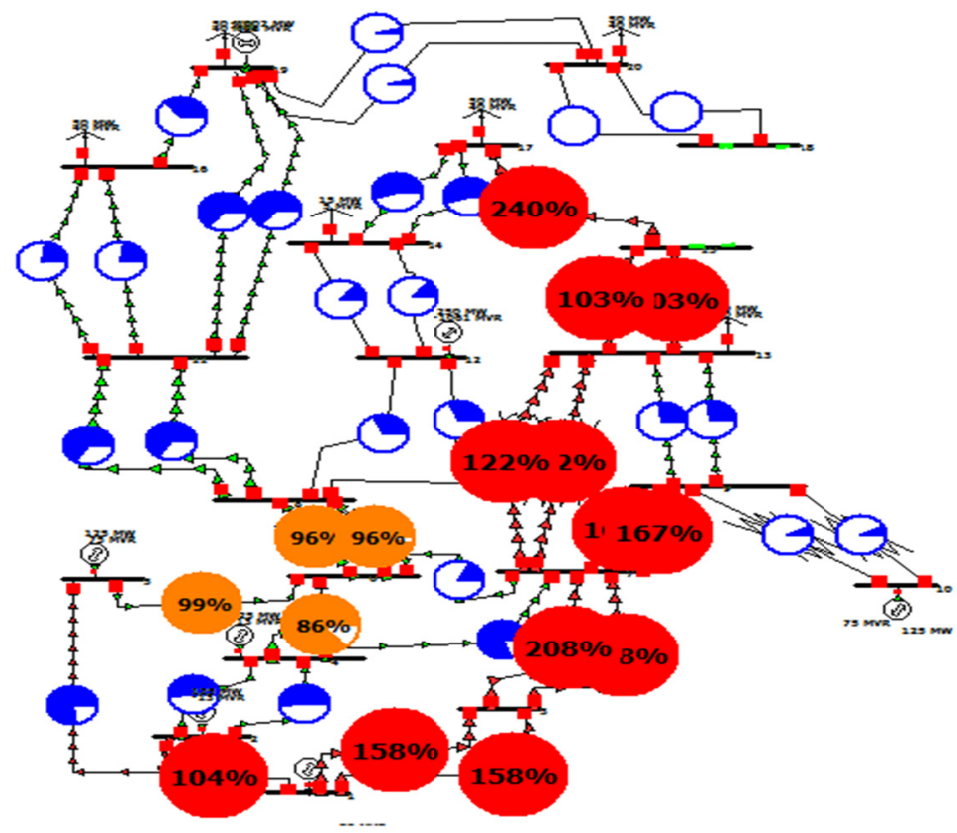

Figure 5. Represents N-2 power flow case

After doing all contingency studies as stated, table 1 was obtained which represent all lines which cannot be out 
of the system in same time.

Some of cases will be addressed to explain the developed method under flowing conditions:

1. The program or the algorithm will find solution to the problem (over load or number of crew) when it occur. This means that if there is no problem in the initial schedule (requested schedule) the program will not do any change even though this schedule is not optimal solution. In other words, this developed method finds optimal solution to the problem when it occurs.

2. The relationship between the tabu search and algorithm is neighbor solutions so that it goes from one candidate solution to another one then choose the optimal solution from the candidates' solutions.

Table 1. The prohibited lines

\begin{tabular}{lllllll}
\hline LINE OFF & EFFECTED & LINE OFF & EFFECTED & LINE OFF & EFFECTED & LINE OFF \\
\hline 1,2 & OVERLOAD & 1,7 & OVERLOAD & 3,7 & OVERLOAD & $9,5,1$ \\
3,4 & OVERLOAD & 1,8 & OVERLOAD & 3,8 & OVERLOAD & $9,5,2$ \\
5,6 & OVERLOAD & 2,7 & OVERLOAD & 4,7 & OVERLOAD & \\
7,8 & OVERLOAD & 2,8 & OVERLOAD & 4,8 & OVERLOAD & \\
9,10 & OVERLOAD & $9,7,5$ & OVERLOAD & $9,5,1$ & OVERLOAD & \\
1,3 & OVERLOAD & $9,7,6$ & OVERLOAD & $1,3,5,7,9$ & OVERLOAD & \\
1,4 & OVERLOAD & $9,8,5$ & OVERLOAD & $10,6,1$ & OVERLOAD & \\
2,4 & OVERLOAD & $10,7,5$ & OVERLOAD & $10,8,5$ & OVERLOAD & \\
2,3 & OVERLOAD & $10,7,6$ & OVERLOAD & $10,8,6$ & OVERLOAD & \\
$10,5,1$ & OVERLOAD & $9,5,1$ & OVERLOAD & $9,6,1$ & OVERLOAD & \\
& & $9,5,2$ & & $9,6,2$ & & \\
\hline
\end{tabular}

\section{Maintenance scheduling for case 1:}

The proposed maintenance schedule for case 1 , given in table 2 is considered as the initial requested schedule by local maintenance centers. It has ten (10) maintenance outage tasks. The schedule proposes to maintain line 1 on Wednesday, line 2 on Friday, line 4 on Tuesday, line 5 on Friday, line 6 on Tuesday, Wednesday and Thursday, line 7 on Monday, line 8 on Saturday and Sunday, line 9 on Saturday, Sunday and Monday and line 10 on Tuesday. The program will generate the base configuration of the system. Also, it will save the given schedule as "the initial requested schedule" and will check for both constraints (overload and number of crews). Table 2 shows that the schedule at Tuesday has overload between tasks 4 and 7, since, taking out line 4 and 7 at same time will generate overload on the system. First step to solve the overload by moving of starting dates. Task no. 4 has higher priority than task no. 7 so that task no.7 will move to resolve the overload problem. The program will search for optimal move between neighbor solutions. The solution (optimal solution) is given in table 3 .

Table 2. Initial schedule for the 20 bus system-case no. 1

\begin{tabular}{|c|c|c|c|c|c|c|c|c|}
\hline$\frac{\tilde{n}}{\tilde{\Xi}}$ & $\cdot \vec{D} \cdot \vec{D}$ & Saturday & Sunday & Monday & $\begin{array}{c}\text { Days } \\
\text { Tuesday }\end{array}$ & Wednesday & Thursday & Friday \\
\hline 1 & 3 & & & & & Line 1 & & \\
\hline 2 & 2 & & & & & & & Line 5 \\
\hline 3 & 1 & & & & & & & \\
\hline 4 & 4 & & & & Line 4 & & & \\
\hline 5 & 5 & & & & & & & Line 2 \\
\hline 6 & 6 & & & & & Line 6 & & \\
\hline 7 & 7 & & & & Line 7 & & & \\
\hline 8 & 8 & Line 8 & & & & & & \\
\hline 9 & 9 & Line 9 & & & & & & \\
\hline 10 & 10 & & & & Line 10 & & & \\
\hline
\end{tabular}


Table 3. Final schedule of case no. 1 obtained after TS program

\begin{tabular}{|c|c|c|c|c|c|c|c|c|}
\hline $\begin{array}{l}\frac{y}{y} \\
\frac{y}{\Xi}\end{array}$ & 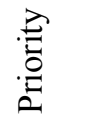 & Saturday & Sunday & Monday & $\begin{array}{c}\text { Days } \\
\text { Tuesday }\end{array}$ & Wednesday & Thursday & Friday \\
\hline 1 & 3 & & & & & Line 1 & & \\
\hline 2 & 2 & & & & & & & Line 5 \\
\hline 3 & 1 & & & & & & & \\
\hline 4 & 4 & & & & Line 4 & & & \\
\hline 5 & 5 & & & & & & & Line 2 \\
\hline 6 & 6 & & & & Line 6 & & & \\
\hline 7 & 7 & & & Line 7 & & & & \\
\hline 8 & 8 & Line 8 & & & & & & \\
\hline 9 & 9 & Line 9 & & & & & & \\
\hline 10 & 10 & & & & Line 10 & & & \\
\hline
\end{tabular}

\section{Maintenance scheduling for case 2:}

The maintenance schedule of case 2 is given in table 4 . There is overload between task number 3,4 and 2 . Tasks number 4 and 2 have a higher priority than task number 3 which means that task number 3 will be moved to remove the overload problem. In same manner as explained in case 1, case number 2 will proceed until the final solution is obtained by the TS program. Table 5 is the final solution for case 2 .

Table 4. Initial schedule for the 20 bus system-case no. 2

\begin{tabular}{|c|c|c|c|c|c|c|c|c|c|}
\hline \multirow{2}{*}{$\begin{array}{l}\frac{\tilde{y}}{\varpi} \\
\Phi\end{array}$} & \multirow{2}{*}{$\stackrel{D}{2}$} & \multicolumn{8}{|c|}{ Days } \\
\hline & & Saturday & & Sunday & Monday & Tuesday & Wednes. & Thursday & Friday \\
\hline 1 & 6 & & & & & & & & Line 1 \\
\hline 2 & 8 & & & Line 2 & & & & & \\
\hline 3 & 9 & & Line 3 & & & & & & \\
\hline 4 & 7 & & Line 4 & & & & & & \\
\hline 5 & 10 & & & Line 5 & & & & & \\
\hline 6 & 4 & & & & & Line 9 & & & \\
\hline 7 & 5 & & & & & & & & \\
\hline 8 & 2 & & & & & & & & \\
\hline 9 & 3 & & & & & Line 6 & & & \\
\hline 10 & 1 & & Line 10 & & & & & & \\
\hline
\end{tabular}

Table 5. Final schedule of case no. 2 obtained after TS program

\begin{tabular}{|c|c|c|c|c|c|c|c|c|}
\hline$\frac{n}{\tilde{a}}$ & $\stackrel{\vec{D}}{\vec{E}} \geq$ & Saturday & Sunday & Monday & $\begin{array}{c}\text { Days } \\
\text { Tuesday }\end{array}$ & Wednes. & Thursday & Friday \\
\hline 1 & 6 & & & & & & & Line 1 \\
\hline 2 & 8 & & & Line 2 & & & & \\
\hline 3 & 9 & & & & & Line 3 & & \\
\hline 4 & 7 & Line 4 & & & & & & \\
\hline 5 & 10 & & & Line 5 & & & & \\
\hline 6 & 4 & & & & & Line 9 & & \\
\hline 7 & 5 & & & & & & & \\
\hline 8 & 2 & & & & & & & \\
\hline 9 & 3 & & & & & Line 6 & & \\
\hline 10 & 1 & Line 10 & & & & & & \\
\hline
\end{tabular}




\section{Maintenance scheduling case 3:}

The proposed schedule, given in table 6, has overload problem in Saturday and Sunday (between task 4 and 8). Based on the priorities of tasks, task number 4 will be shifted to remove the overload. Moving these task, introduces a new overload in the system in Monday (between task 4 and 7). The program will try to solve this by move task number 4 which is less priority until this problem is solved. The staring date of task number 4 is Wednesday. The table 7 is the final solution of case 3 .

Table 6. Initial schedule for the 20 bus system- case no. 3

\begin{tabular}{|c|c|c|c|c|c|c|c|c|}
\hline$\frac{n}{\frac{n}{\pi}}$ & 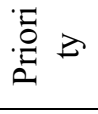 & Saturday & Sunday & Monday & $\begin{array}{c}\text { Days } \\
\text { Tuesday }\end{array}$ & Wednes. & Thursday & Friday \\
\hline 1 & 1 & & & & & & & \\
\hline 2 & 2 & & & & & & & \\
\hline 3 & 3 & & & & & Line 9 & & \\
\hline 4 & 10 & Line 4 & & & & & & \\
\hline 5 & 5 & Line 5 & & & & & & \\
\hline 6 & 6 & & & Line 6 & & & & \\
\hline 7 & 7 & & & Line 7 & & & & \\
\hline 8 & 8 & Line 8 & & & & & & \\
\hline 9 & 2 & & & & & Line 3 & & \\
\hline 10 & 4 & & & & Line 10 & & & \\
\hline
\end{tabular}

Table 7. Final schedule of case no. 3 obtained after TS program

\begin{tabular}{|c|c|c|c|c|c|c|c|c|}
\hline$\frac{n}{\frac{n}{5}}$ & $\stackrel{\square}{0}$ & Saturday & Sunday & Monday & $\begin{array}{c}\text { Days } \\
\text { Tuesday }\end{array}$ & Wednes. & Thursday & Friday \\
\hline 1 & 1 & & & & & & & \\
\hline 2 & 2 & & & & & & & \\
\hline 3 & 3 & & & & & Line 9 & & \\
\hline 4 & 10 & & & & Line 4 & & & \\
\hline 5 & 5 & Line & & & & & & \\
\hline 6 & 6 & & & Line 6 & & & & \\
\hline 7 & 7 & & & Line 7 & & & & \\
\hline 8 & 8 & Line & & & & & & \\
\hline 9 & 2 & & & & & Line 3 & & \\
\hline 10 & 4 & & & & Line 10 & & & \\
\hline
\end{tabular}

\section{Maintenance scheduling case no. 4:}

The initial requested maintenance schedule of case 4 is given in table 8 . The schedule has no overload problems and crews conflict. Since the proposed schedule has no overload and has no crew's conflict, no moves of starting dates are required. Therefore, the output is the same as the initial requested schedule since no moves were encountered. The final solution of case 4 is given in table 9 . 
Table 8 . Initial schedule for the 20 bus system- case no. 4

\begin{tabular}{|c|c|c|c|c|c|c|c|c|}
\hline$\frac{\mathscr{y}}{\tilde{E}}$ & $\stackrel{\overline{0}}{\overrightarrow{0}} \underset{2}{2}$ & Saturday & Sunday & Monday & $\begin{array}{c}\text { Days } \\
\text { Tuesday }\end{array}$ & Wednes. & Thursday & Friday \\
\hline 1 & 1 & & & & & & & \\
\hline 2 & 3 & & & & & & & \\
\hline 3 & 5 & & & & & Line 6 & & \\
\hline 4 & 7 & Line 5 & & & & & & \\
\hline 5 & 9 & Line 4 & & & & & & \\
\hline 6 & 2 & & & & & Line 3 & & \\
\hline 7 & 4 & & & & & & & \\
\hline 8 & 6 & & & Line 8 & & & & \\
\hline 9 & 8 & Line 9 & & & & & & \\
\hline 10 & 10 & & & & Line 10 & & & \\
\hline
\end{tabular}

Table 9. Final schedule of case no. 4 obtained after TS program

\begin{tabular}{|c|c|c|c|c|c|c|c|c|}
\hline$\frac{n}{\tilde{n}}$ & 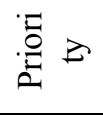 & Saturday & Sunday & Monday & $\begin{array}{c}\text { Days } \\
\text { Tuesday }\end{array}$ & Wednesday & Thursday & Friday \\
\hline 1 & 1 & & & & & & & \\
\hline 2 & 3 & & & & & & & \\
\hline 3 & 5 & & & & & Line 6 & & \\
\hline 4 & 7 & Line 5 & & & & & & \\
\hline 5 & 9 & Line 4 & & & & & & \\
\hline 6 & 2 & & & & & Line 3 & & \\
\hline 7 & 4 & & & & & & & \\
\hline 8 & 6 & & & Line 8 & & & & \\
\hline 9 & 8 & Line 9 & & & & & & \\
\hline 10 & 10 & & & & Line 10 & & & \\
\hline
\end{tabular}

\section{Conclusion}

The developed scheduling methods were applied to 20-Bus test system and from the results, it shows the following:

1. It is confirmed that the developed methods are useful and practical as scheduling tools.

2. The works progress is maximized in the scheduling period while minimized the carry-over work to the next scheduling period.

3. The deviation of date work (starting of the work) which could be requested from local maintenance centers is minimized.

4. The results have been obtained in low execution times.

5. It shows a good performance for functions having small number of variables.

6. Easy to implement.

\section{Acknowledgements}

The authors gratefully acknowledge the contributions of Dr. Noaman M. Noaman for their work on the original version of this document.

\section{References}

Alshaikh, A., \& El-Amin, I. (2001). Automatic scheduling of substation maintenance outages. MS dissertation department of graduate studies university of KFUPM (pp. 1-20).

Furukawa, T., Nomoto, M., Nagasawa, T., Sasaki, T., Deno, K., \& Maekawa, T. (1991). Automatic scheduling method using tabu search for maintenance outages tasks of transmission and substation system with network constraints. Journal of Power Engineering Society, 895-900. 
Glover, F. (1989). Tabu search - part I. Operations research society of America, 1, 190-206.

Glover, F., Taillard, E., \& Werra, D. (1993). Auser's guide to tabu search. Netherlands. Retrieved from http://smartgrid.epri.com/UseCases/ContingencyAnalysis-Baseline.pdf.

Reihani, E., Davodi, M., Najjar, M., \& Norouzizadeh, R. (2010). Reliability based generator maintenance scheduling using hybrid evolutionary approach. Energy Conference and Exhibition (EnergyCon), 847-852.

Stagg, G., \& El-Abiad, A. (1968). Computer methods I power system analysis. McGraw-Hill, New York.

Sawa, T. (1999). Automatic scheduling method using tabu search for maintenance outages tasks of transmission and substation system with network constraints. Journal of Power Engineering Society, 2, 895-900.

Tanaka, H., Magori, H., Niimura, T., \& Yokoyama, R. (2010). Optimal replacement scheduling of obsolete substation equipment by branch \& bound method. Journal of Power and Energy, 1-6.

Yang, F. (2009). Multiobjective evolutionary optimization of maintenance schedules and extents for composite power systems. Journal of Power System, 4, 1694-1702. http://dx.doi.org/10.1109/TPWRS.2009.2030354

Yang, F. (2009). Optimisation of maintenance schedules and extents for composite power systems using multi-objective evolutionary algorithm. Journal of generation, transmission \& distribution, 10, 930-940. http://dx.doi.org/10.1049/iet-gtd.2009.0172 